\title{
Feasibility of In Situ, High-Resolution Correlation of Tracer Uptake with Histopathology by Quantitative Autoradiography of Biopsy Specimens Obtained Under ${ }^{18}$ F-FDG PET/CT Guidance
}

\author{
Louise M. Fanchon ${ }^{1,2}$, Snjezana Dogan ${ }^{3}$, Andre L. Moreira ${ }^{3}$, Sean A. Carlin ${ }^{4}$, C. Ross Schmidtlein ${ }^{1}$, Ellen Yorke ${ }^{1}$, \\ Aditya P. Apte ${ }^{1}$, Irene A. Burger ${ }^{5}$, Jeremy C. Durack ${ }^{4}$, Joseph P. Erinjeri ${ }^{4}$, Majid Maybody ${ }^{4}$, Heiko Schöder ${ }^{4}$, \\ Robert H. Siegelbaum ${ }^{4}$, Constantinos T. Sofocleous ${ }^{4}$, Joseph O. Deasy ${ }^{1}$, Stephen B. Solomon ${ }^{4}$, John L. Humm ${ }^{1}$, \\ and Assen S. Kirov ${ }^{1}$ \\ ${ }^{I}$ Department of Medical Physics, Memorial Sloan-Kettering Cancer Center, New York, New York; ${ }^{2}$ INSERM, UMR1101, LaTIM, \\ Brest, France; ${ }^{3}$ Department of Pathology, Memorial Sloan-Kettering Cancer Center, New York, New York; ${ }^{4}$ Department of Radiology, \\ Memorial Sloan-Kettering Cancer Center, New York, New York; and ${ }^{5}$ Department of Nuclear Medicine, University Hospital, Zurich, \\ Switzerland
}

Core biopsies obtained using PET/CT guidance contain bound radiotracer and therefore provide information about tracer uptake in situ. Our goal was to develop a method for quantitative autoradiography of biopsy specimens (QABS), to use this method to correlate ${ }^{18} \mathrm{~F}-\mathrm{FDG}$ tracer uptake in situ with histopathology findings, and to briefly discuss its potential application. Methods: Twenty-seven patients referred for a PET/CT-guided biopsy of ${ }^{18} \mathrm{~F}-\mathrm{FDG}$-avid primary or metastatic lesions in different locations consented to participate in this institutional review board-approved study, which complied with the Health Insurance Portability and Accountability Act. Autoradiography of biopsy specimens obtained using 5 types of needles was performed immediately after extraction. The response of autoradiography imaging plates was calibrated using dummy specimens with known activity obtained using 2 core-biopsy needle sizes. The calibration curves were used to quantify the activity along biopsy specimens obtained with these 2 needles and to calculate the standardized uptake value, SUV $\mathrm{V}_{\mathrm{ARG}}$. Autoradiography images were correlated with histopathologic findings and fused with PET/CT images demonstrating the position of the biopsy needle within the lesion. Logistic regression analysis was performed to search for an SUV $V_{\text {ARG }}$ threshold distinguishing benign from malignant tissue in liver biopsy specimens. Pearson correlation between $S_{U} V_{A R G}$ of the whole biopsy specimen and average SUV $V_{\mathrm{PET}}$ over the voxels intersected by the needle in the fused PET/CT image was calculated. Results: Activity concentrations were obtained using autoradiography for 20 specimens extracted with 18- and 20-gauge needles. The probability of finding malignancy in a specimen is greater than $50 \%$ (95\% confi-


served shape and orientation and in the absence of motion, one can achieve autoradiography, CT, and PET image registration with spatial accuracy better than $2 \mathrm{~mm}$. The correlation coefficient between the mean specimen SUV $V_{A R G}$ and $S V_{P E T}$ was 0.66. Conclusion: Performing QABS on core-biopsy specimens obtained using PET/CT

Received Sep. 17, 2014; revision accepted Jan. 26, 2015.

For correspondence or reprints contact: Assen S. Kirov, Department of Medical Physics, Memorial Sloan-Kettering Cancer Center, 1275 York Ave., New York, NY 10021.

E-mail: kirova@mskcc.org

Published online Feb. 26, 2015

COPYRIGHT (C) 2015 by the Society of Nuclear Medicine and Molecular Imaging, Inc. guidance enables in situ correlation of ${ }^{18} \mathrm{~F}-\mathrm{FDG}$ tracer uptake and histopathology on a millimeter scale. QABS promises to provide useful information for guiding interventional radiology procedures and localized therapies and for in situ high-spatial-resolution validation of radiopharmaceutical uptake.

Key Words: autoradiography; biopsy; PET/CT; histopathology; oncology

J Nucl Med 2015; 56:538-544

DOI: 10.2967/jnumed.114.148668

$\mathbf{I}_{\mathrm{n}}$ the last decade, the use of PET/CT imaging to localize metabolically active lesions has increased for the purpose of diagnosis and staging as well as for image-guided biopsies and tumor ablations. Accurate determination of cancer margins is essential for the success of local treatment strategies such as radiotherapy or thermal ablative therapies (1), and PET/CT has been shown to play an important role (2-4). However, despite the advantages of molecular cancer imaging, the value of PET for defining tumor boundaries is limited by low resolution and the lack of standardized windowing protocols. In addition, other physical, technical, and biologic factors introduce uncertainties in quantification of PET activity and tumor segmentation $(5,6)$. The purpose for this study was to evaluate autoradiography as a tool for high-spatial-resolution assessment of metabolic activity in biopsy samples relative to histopathology.

PET-histopathology correlations have been investigated previously for several tumor types as reviewed elsewhere (7). Most of these studies were performed to evaluate PET image segmentation for radiation therapy, where accurate delineation of the tumor boundary is essential. However, the time difference between the scan and the tumor excision, as well as specimen deformation during the fixation process, introduce spatial inaccuracies. Also, because of variations in patient anatomy, in scanners, and in imaging protocols, results from these investigations cannot be easily translated to other institutions and patients. Correlating specimen histopathology and tracer uptake as determined from autoradiography with the PET/CT images 
provides information that can help determine the gross tumor volume for future treatments of the same patient.

Image-guided needle biopsies are critical for diagnosis and management in oncology (8). The use of PET/CT guidance is of growing importance for interventional procedures, and several studies (9-13) have reported that PET/CT guidance has potential for improving targeting and diagnostic efficiency. It has also been shown that having an onsite cytotechnologist to evaluate specimen adequacy improves biopsy efficacy and patient care $(14,15)$. These evaluations may reduce complications by avoiding unnecessary needle punctures. Because having an on-site cytotechnologist is often limited to large institutions, we are investigating an alternative method to cytopathology for specimen evaluation of adequacy in PET/CT-guided procedures.

The presence of radioactive tracer in the specimen allows highresolution analysis of its distribution along the tissue sample. The tracer distribution can then be spatially correlated with the histologic sections and the PET/CT images, which may improve the accuracy of PET-guided interventions (9-11). Because ${ }^{18} \mathrm{~F}-\mathrm{FDG}$ uptake correlates with the number of viable tumor cells (16), in this study autoradiography was used to measure radioactivity for iden- tification of malignancy at the time of specimen acquisition. We describe the correlation between our initial quantitative autoradiography findings, biopsy sample histopathology, and PET imaging, as well as the potential impact of these techniques.

\section{MATERIALS AND METHODS}

\section{Patients}

Informed consent was obtained from all patients $(n=27)$ in this institutional review board-approved (approval 12-072) prospective study, which complied with the Health Insurance Portability and Accountability Act. Patients referred for PET/CT-guided biopsies between May 2012 and October 2014 independent of tumor type and location were considered. PET/CT-guided biopsies are performed at our institution for metabolically active lesions that are not well visualized using other imaging modalities. Biopsy specimens taken with different devices were evaluated, including 11-, 13-, 18-, 20-gauge core needles and 22-gauge fine aspiration needles. Early in the process it was determined that 18- and 20-gauge core needles (Temno Evolution, Inc., inner diameters of 0.84 and $0.6 \mathrm{~mm}$, respectively, and 20-mm-long specimen bed), giving relatively longer and more contiguous

TABLE 1

Biopsy Results for Enrolled Patients

\begin{tabular}{|c|c|c|c|}
\hline Patient no. & Gauge & Lesion location & Pathologic finding \\
\hline 1 & 11 & Iliac bone & $\begin{array}{l}\text { Blood clot and fragments of cellular marrow showing } \\
\text { maturing hematopoietic cells }\end{array}$ \\
\hline 2 & 18 & Iliac node & Hodgkin lymphoma \\
\hline 3 & 18 & Mesenteric node & Atypical histiocytic infiltrate \\
\hline 4 & 11 & Acetabulum & Metastatic squamous cell carcinoma \\
\hline 5 & 20 & Liver & Metastatic adenocarcinoma \\
\hline 6 & 22 & Pelvis & Scant fragments of atypical glandular epithelium \\
\hline 7 & 18 & Liver & Serous carcinoma \\
\hline 8 & 18 & Liver & Metastatic adenocarcinoma \\
\hline 9 & 18 & Pelvis & Plasma cell neoplasm \\
\hline 10 & 20 & Liver & Metastatic adenocarcinoma \\
\hline 11 & 13 & Scapula & Metastatic carcinoma \\
\hline 12 & 18 & Liver & Metastatic adenocarcinoma \\
\hline 13 & 18 & Liver & Metastatic adenocarcinoma \\
\hline 14 & 18 & Liver & Metastatic adenocarcinoma \\
\hline 15 & 18 & Scapula & Diffuse large B-cell lymphoma \\
\hline 16 & 18 & Liver & Metastatic adenocarcinoma \\
\hline 17 & 18 & Liver & Metastatic adenocarcinoma \\
\hline 18 & 18 & Liver & Metastatic adenocarcinoma \\
\hline 19 & 18 & Liver & Metastatic adenocarcinoma \\
\hline 20 & 18 & Liver & Metastatic adenocarcinoma \\
\hline 21 & 18 & Liver & Metastatic adenocarcinoma \\
\hline 22 & 18 & Leg muscle & $\begin{array}{l}\text { Skeletal muscle with degenerative and reactive changes } \\
\text { consistent with treatment-related abnormalities }\end{array}$ \\
\hline 23 & 18 & Lung & Non-small cell carcinoma \\
\hline 24 & 18 & Liver & Poorly differentiated adenocarcinoma \\
\hline 25 & 18 & Liver & Poorly differentiated carcinoma \\
\hline 26 & 18 & Liver & Metastatic adenocarcinoma \\
\hline 27 & 18 & Liver & Metastatic carcinoma \\
\hline
\end{tabular}




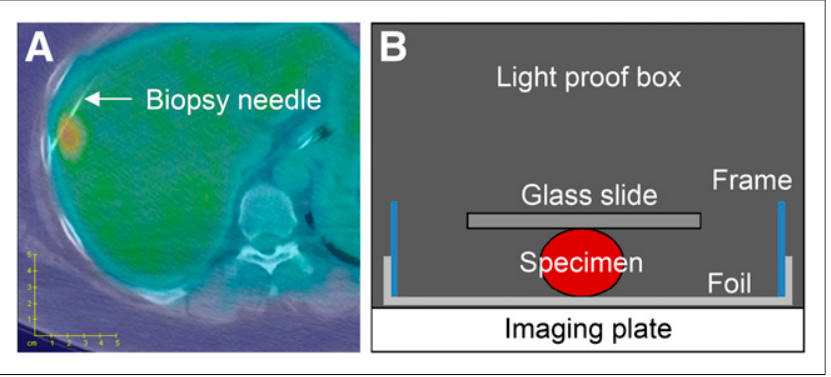

FIGURE 1. (A) Axial ${ }^{18}$ F-FDG PET image fused with a CT image with biopsy needle in place (patient 20). (B) Specimen autoradiography schematic.

specimens, were most suitable for this protocol. All subsequent cases were performed using these 2 needles. Table 1 summarizes patient data, including disease location, needle size, and histopathologic diagnosis.

\section{PET/CT-Guided Biopsies}

Each biopsy was performed in a dedicated interventional radiology suite equipped with a D690 PET/CT scanner (GE Healthcare). Patients were intravenously injected with a lower dose of ${ }^{18} \mathrm{~F}$-FDG than is standard for diagnostic imaging in accordance with previously described interventional protocols $(222 \mathrm{MBq} \pm 10 \%$ for biopsies and $166 \mathrm{MBq} \pm 10 \%$ for biopsies before ablation) (17). PET-avid lesions were well visualized even more than $3 \mathrm{~h}$ after injection. The average uptake period was 107 min (range, 62$186 \mathrm{~min}$ ). Procedures were performed with patients under moderate sedation, under monitored anesthesia care, or under general anesthesia based on comorbidities, anticipated procedure length, and procedural risk assessment in consultation with the anesthesiology service. An initial PET scan (5 min/ bed position) and CT scan were acquired for biopsy targeting. With the needle in position to acquire tissue from the target lesion, a 1-bed-position CT scan was acquired and fused with the initial PET scan (Fig. 1A). Each specimen extracted was evaluated by a cytotechnologist using a touch preparation technique on a glass slide. Typically, between 2 and 6 specimens were extracted from each patient by experienced interventional radiologists until the obtained tissue was considered adequate for diagnosis. The PET scans were reconstructed using VuePointHD software (GE Healthcare) with time-of-flight and SharpIR resolution correction turned on, and using the "Heavy" $z$-axis filter and a gaussian transaxial filter of $6.4 \mathrm{~mm}$ in full width at half maximum, with 2 iterations and 16 subsets. The PET intrinsic resolution varies between 4.6 and $6.5 \mathrm{~mm}$ and is expected to be slightly poorer under clinical conditions. PET images were upsampled from $128 \times 128$ or $256 \times 256$ to $512 \times 512$ $(1.35 \times 1.35 \times 3.27 \mathrm{~mm}$ voxels $)$ for further analysis.

\section{Quantitative Autoradiography of Biopsy Specimens (QABS)}

For each case, autoradiography (Fig. 1B) was performed on a single contiguous specimen longer than $0.5 \mathrm{~cm}$. Immediately after extraction, the specimen was placed on a glass slide and its orientation within the needle was marked with ink (Fig. 2B). The specimen was sprayed with a solution that sets the tissue-marking dye (Ink Aid; Cancer Diagnostic Inc.), and saline was added to protect the specimen from drying out. Photos of the specimen were taken: in the needle, on the slide before and after marking, and through the slide when placed on thin $(0.01 \mathrm{~mm})$ plastic foil stretched on a frame. Subsequently, the frame with the specimen was placed on an autoradiography imaging plate (Fujifilm BAS-MS 2025) (Fig. 1B). The plate was exposed to the specimen for $10 \mathrm{~min}$ in a dark enclosure to prevent exposure to ambient light. After the first 2 cases, the autoradiography system was moved just outside the interventional suite to eliminate scattered photons from the CT scan and the patient.

The total time for specimen photography and autoradiography was about $15 \mathrm{~min}$; thereafter, the specimens were placed in formalin and transported to the pathology department according to the usual standard of care. The plate was then scanned using a Typhoon FLA 7000 laser scanner (GE Healthcare) $20 \mathrm{~min}$ after the exposure, and the data were stored in a 16-bit gray-scale image.

The autoradiography plate was calibrated using surrogate specimens with a known activity concentration. A gel was prepared from gelatin $(5 \mathrm{~g})$, glycerol $\left(30 \mathrm{~g}\right.$ ), water $(15 \mathrm{~g})$, and ${ }^{18} \mathrm{~F}-\mathrm{FDG}$ (typically $18.5 \mathrm{MBq}$ in $1 \mathrm{~mL}$ ) and withdrawn using 20- and 18-gauge core needles to obtain surrogate specimens with dimensions similar to those of the clinical samples.

Autoradiography images of these surrogate specimens were acquired for activities decaying from 130 to $0.7 \mathrm{kBq} / \mathrm{g}$, following the same exposure-readout protocol as for clinical cases. The measurement was repeated 3 times using 2 specimens for each needle size. The autoradiograph was segmented using contours drawn on the specimen's photo, and the mean number of counts per pixel was plotted as a function of activity concentration in the gelatin specimen at the time of exposure (Fig. 3).

Using these calibration curves, the activity concentration of the biopsy specimens extracted from 20 patients using the 18- or 20-gauge needle was assessed. $\mathrm{SUV}_{\mathrm{ARG}}$ was calculated using the following formula:

$$
\mathrm{SUV}_{\mathrm{ARG}}=\frac{A_{\text {spec }}}{A_{\text {inj }} / W}
$$

where $A_{\text {spec }}$ is the specimen activity as determined using the calibration curves (Fig. 3), $A_{i n j}$ is the injected activity (both decay-corrected to the time of the PET scan), and $W$ is the weight of the patient.

The resolution of the autoradiography images was estimated using a gaussian function model of the point-spread function. The gaussian parameters were optimized by comparing the autoradiography images to the convolution of the model function with a mask obtained from a photograph of 18- and 20-gauge surrogate specimens.

\section{Histopathology}

Specimens were embedded in paraffin, and 4- $\mu \mathrm{m}$-thick sections were prepared and analyzed after hematoxylin-eosin staining by experienced pathologists. Micrographs of the largest cross-sectional slice containing

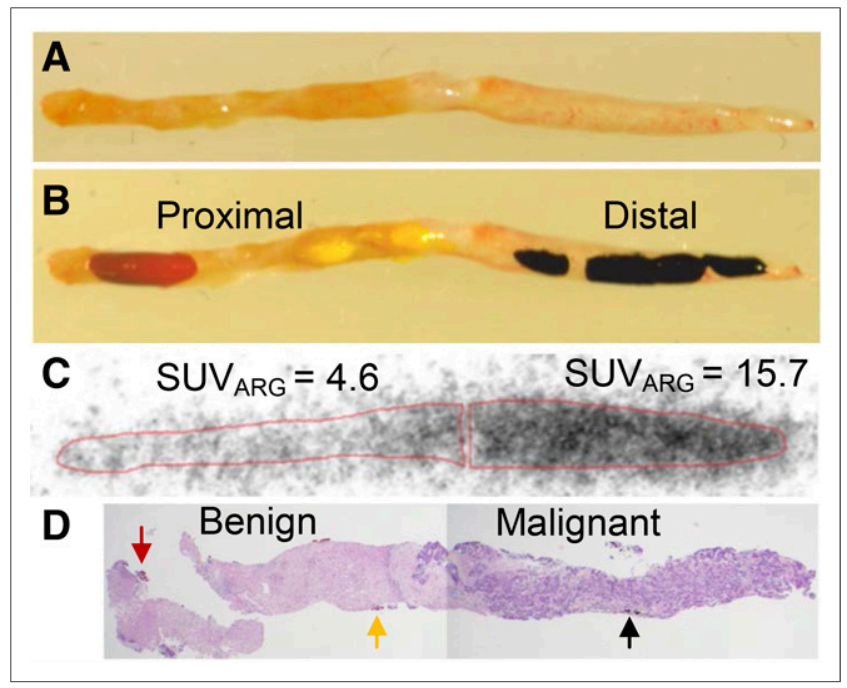

FIGURE 2. (A and B) Photographs of biopsy specimen (patient 20) before (A) and after marking with red, yellow, and black ink (B). (C and D) Corresponding autoradiograph (C) and histopathology slide (D). Colored arrows point to areas on micrograph where the ink marks are visible. Average $S_{\text {SU }} V_{A R G}$ for contoured regions of autoradiography image (C) are quoted. 


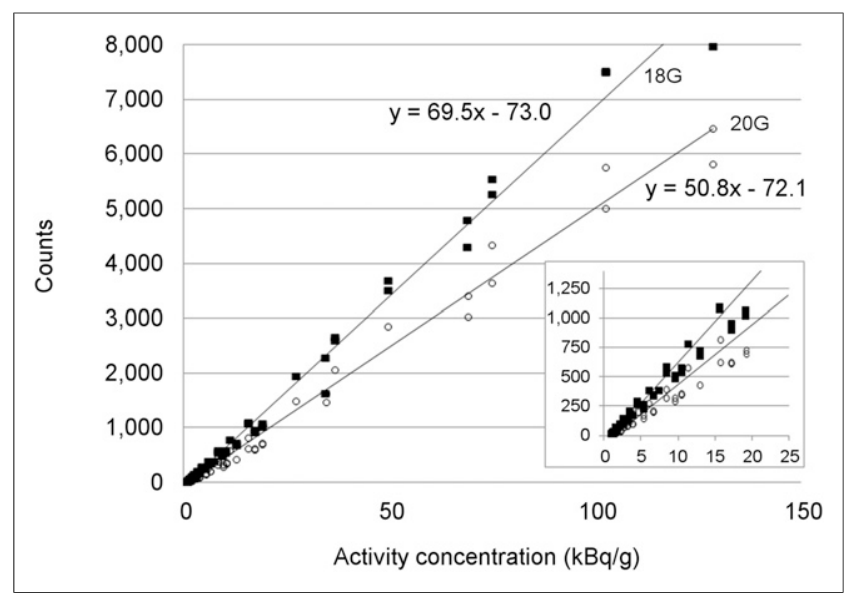

FIGURE 3. Calibration curves for 18- and 20-gauge core-needle biopsy specimens.

visible ink marks were studied for correlation with the specimen photo and the autoradiography image.

\section{Image Registration and Correlations}

Autoradiography and Histopathology. Patients positive for carcinoma $(n=2)$ or adenocarcinoma $(n=12)$ metastatic to the liver were included in this analysis. The micrograph was visually registered with the photo, with the help of the ink marks (Figs. 2B and 2D). A pathologist indicated benign and malignant regions on the micrograph. Contours delineating those regions were then drawn on the photo and used to segment the autoradiography. For homogeneous specimens (either entirely benign or malignant), the mean $\mathrm{SUV}_{\mathrm{ARG}}$ of the whole specimen was calculated. For heterogeneous specimens (presence of both benign and malignant tissue), $\mathrm{SUV}_{\mathrm{ARG}}^{\text {benign }}$ and $\mathrm{SUV}_{\mathrm{ARG}}^{\text {malignant }}$ were calculated (Fig. 2C). The correlation between presence or absence of malignant cells and $\mathrm{SUV}_{\mathrm{ARG}}$ was analyzed using logistic regression. To evaluate the uncertainty of $\mathrm{SUV}_{\mathrm{ARG}}$, it was calculated several times for each region by shifting the contours within the photograph-autoradiograph registration uncertainty and the autoradiograph resolution uncertainty.

Autoradiography and PET. Autoradiography images were aligned with the needle track on the CT images using the open-source Computational Environment for Radiation Research software (18). The autoradiography image was superimposed on the corresponding slice of the PET image of the PET/CT scan (Fig. 4). The mean $\mathrm{SUV}_{\mathrm{PET}}$ over the upsampled voxels $(1.37 \times 1.37 \times 3.27 \mathrm{~mm})$ intersected by the needle was compared with the $\mathrm{SUV}_{\mathrm{ARG}}$ of the whole specimen.

\section{RESULTS}

\section{Autoradiography}

Biopsy specimen handling, ink marking, and autoradiography did not adversely affect routine histopathologic analysis. Exposure of the imaging plates for $10 \mathrm{~min}$ proved to be sufficient for imaging specimen activities down to about $1 \mathrm{kBq} / \mathrm{g}$. Autoradiography images with detectable signal were obtained for 24 of the 27 specimens. Autoradiography images could not be obtained for the specimens of 3 patients: in patient 2 because of saturation of the plate from scattered CT x-rays; in patient 8 because of malfunction of the autoradiography plate scanner; and in patient 13 because of low ${ }^{18} \mathrm{~F}$ FDG uptake in a specimen extracted $4 \mathrm{~h}$ after injection.

Specimens extracted with 11-gauge, 13-gauge, and fine aspiration needles presented challenges for the autoradiography technique.
The larger thickness of specimens from 11- and 13-gauge needles (about $2 \mathrm{~mm}$, compared with the average positron range in water: $0.6 \mathrm{~mm}$ (19)) or the presence of bone in the sample interfered with activity calculations. The shape of fine-needle aspiration specimens was too unpredictable to calibrate the autoradiography response. For these reasons, only specimens from 18- and 20-gauge core needles were considered for quantitative analysis.

\section{Calibration Curve and Quantification Accuracy}

The curves plotted in Figure 3 combine the data from all calibration measurements and show that the autoradiography calibration depends on the thickness of the specimen, which depends on the needle size. Since the autoradiography signal comes from the detection of positrons reaching the imaging plate from different depths, specimen thickness affects the signal intensity. Therefore, a separate calibration curve is needed for each needle size. The quantification accuracy of QABS is limited by the precision of the calibration curve, by variations of the specimen thicknesses, and by potential loss of extracellular activity due to smearing by blood or by the added saline. The spread of data points in Figure 3 gives a precision of about $2.5 \mathrm{kBq} / \mathrm{g}$ in the middle of the range of observed biopsy specimen activities $(0-25 \mathrm{kBq} / \mathrm{g})$.

\section{Correlations}

Histopathologically confirmed malignancy is compared with $\mathrm{SUV}_{\mathrm{ARG}}$ in Table 2 and visualized in Figure 5. For 13 patients found positive for metastatic adenocarcinoma or carcinoma to the liver, all specimen segments with a high $\mathrm{SUV}_{\mathrm{ARG}}$ correlated with the presence of malignant cells and segments with a lower $\mathrm{SUV}_{\mathrm{ARG}}$ correlated with benign cells (Figs. 2C, 2D, and 4B). Only 1 specimen had a malignant finding (patient 24) but a low SUV in both the autoradiography and PET images. The separation between benign and malignant tissue is significant (unpaired, 2-sided, nonparametric $t$ test

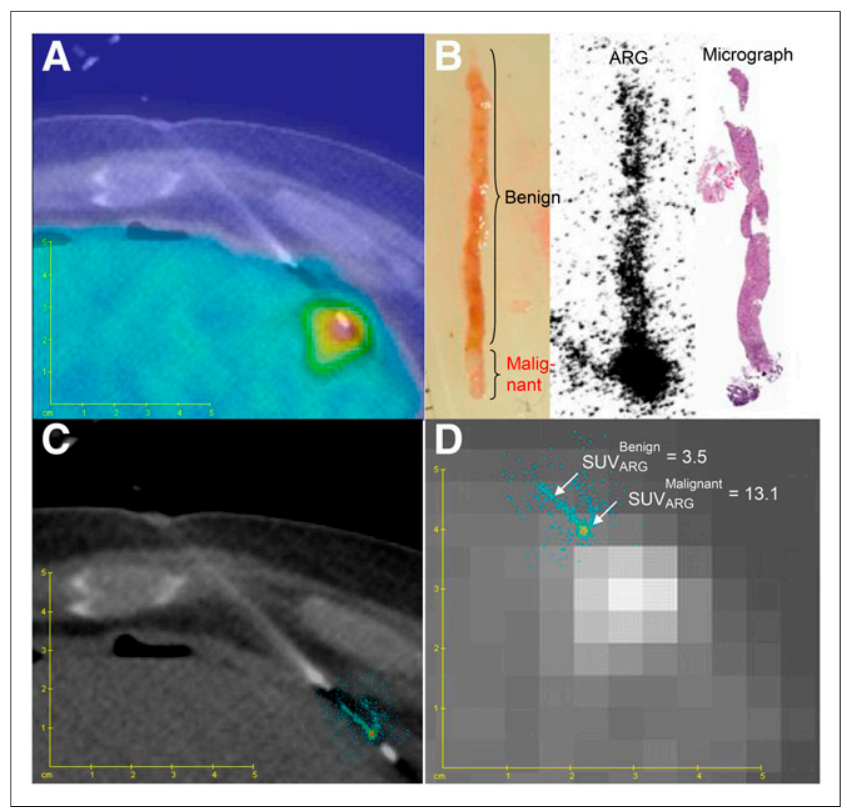

FIGURE 4. (A) Fused PET/CT image for case specifically selected to show peripheral needle placement with both malignant and benign tissue (patient 14). (B) Extracted specimen photograph, autoradiograph, and micrograph. (C and D) Fusion of the autoradiograph with the CT (C) and the PET (D) images. 
TABLE 2

Histopathology and SUV ARG $_{\text {for Liver Biopsy Specimens }}$

\begin{tabular}{|c|c|c|c|}
\hline Histopathology & SUV $_{\text {ARG }}$ & SD & Patient no. \\
\hline \multirow[t]{11}{*}{ Benign } & 1.9 & 0.2 & 21 \\
\hline & 2.0 & 0 & 24 \\
\hline & 2.0 & 0.2 & 12 \\
\hline & 2.4 & 0.2 & 16 \\
\hline & 2.7 & 0 & 17 \\
\hline & 2.9 & 0.1 & 25 \\
\hline & 3.4 & 0.5 & 19 \\
\hline & 3.4 & 0.2 & 27 \\
\hline & 3.5 & 0.1 & 14 \\
\hline & 4.6 & 1.1 & 20 \\
\hline & 5.7 & 0 & 26 \\
\hline \multirow[t]{8}{*}{ Malignant } & 2.4 & 0 & 24 \\
\hline & 7.4 & 0.3 & 19 \\
\hline & 8.6 & 2.3 & 18 \\
\hline & 9.8 & 0.6 & 5 \\
\hline & 10.9 & 1.5 & 27 \\
\hline & 13.1 & 0.1 & 14 \\
\hline & 15.5 & 1.6 & 10 \\
\hline & 15.7 & 0.5 & 20 \\
\hline
\end{tabular}

$P$ value of $1.2 \times 10^{-4}$ ). The probability of finding malignancy in a specimen is greater than $50 \%$ (95\% confidence) if $\mathrm{SUV}_{\mathrm{ARG}}$ is larger than 7.3 (Fig. 5).

Fusion of an autoradiography image with a PET image is shown in Figure 4D. A resolution limit of approximately $1 \mathrm{~mm}$ (full width at half maximum) was determined for autoradiography images of specimens extracted with 18 - and 20-gauge core needles. The overall spatial accuracy for fusing autoradiography images with CT images after accounting for the registration uncertainty is about $1.8 \mathrm{~mm}$ (quadrature sum of $0.5 \mathrm{~mm}$ in half width at half maximum for autoradiography resolution, $1.37 \mathrm{~mm}[=1 \mathrm{CT}$ voxel $]$ and $\sim 1 \mathrm{~mm}$ because of the CT image gray-scale effect on registration) and will be similar for fusion of autoradiography with PET if the registration between CT and PET is not affected by motion.

Comparison of the $\mathrm{SUV}_{\mathrm{ARG}}$ of the whole specimen and average $S_{\text {SET }}$ over the upsampled voxels intersected by the needle in the fused PET/CT image is given in Table 3 and plotted in Figure 6. The Pearson correlation coefficient between $\mathrm{SUV}_{\mathrm{ARG}}$ and $\mathrm{SUV}_{\mathrm{PET}}$ is 0.66. Deviation from a perfect correlation is an expected consequence of the much smaller diameter of the biopsy specimens relative to a PET voxel. The PET voxel represents a single average ${ }^{18}$ F-FDG intensity of the underlying tissue elements, of which the biopsy is a microsample. This deviation is further compounded by possible misregistration due to motion between the PET and CT scans.

\section{DISCUSSION}

The first benefit of the QABS method is its improved spatial resolution compared with clinical PET imaging. Because of the high resolution of the imaging plates $(\sim 25 \mu \mathrm{m})$, the resolution of the QABS method for ${ }^{18} \mathrm{~F}$ PET tracers for correlations between histopathology and the autoradiography image is limited mainly by the positron range and the specimen thickness. If the specimen shape is preserved or can be recovered, correlation of in situ histopathology with ${ }^{18} \mathrm{~F}$-FDG uptake with precision of about $1 \mathrm{~mm}$ is obtainable.

The second major benefit of the method is the ability to assign activity and SUV to tissues with such high resolution. Despite uncertainties in specimen thickness, registration, accuracy of the calibration curve, and smearing of extracellular activity, the method has sufficient precision to clearly separate benign from malignant tissue. Before clinical use for biopsy guidance, further confidence in the $\mathrm{SUV}_{\mathrm{ARG}}$ threshold needs to be built through studying more cases and further investigating the accuracy-limiting factors already mentioned. Such work may allow the use of QABS as an alternative to cytologic evaluation of the specimens.

To translate this method to the clinic, the possibility of displaying the autoradiography image in real time was investigated. Gelatin biopsy specimens were imaged on a Micro Imager (Biospace Lab, S.A.) - a digital detector based on an image-intensifier chargecoupled device-giving real-time images with similar resolution. Use of a similar real-time device dedicated to QABS with increased sensitivity would permit automatic comparison of the activity in the specimen to that seen in the PET image.

The specimen activity calibration curves are valid only for the described experimental procedure. Repetition of the measurements by others would require that calibration curves be built for their specific materials, devices, and protocols. The $\mathrm{SUV}_{\mathrm{ARG}}$ threshold, is dependent on the calibration procedure and gelatin mixture, is intended for biopsy evaluation, and cannot be used for PET image segmentation.

Validating PET images and PET segmentation contours with the pathologic findings from surgically excised lesions can provide histopathologic information for the entire lesion and its boundary. Accurate registration between pathology and PET is, however, often prevented by deformation of the excised specimens and by the poor spatial resolution of PET. One of the recent investigations based on laryngeal lesion excision (20) reports a 3.3-mm registration inaccuracy between the specimen and PET "in the cartilage skeleton." This estimate does not account for possible changes in tumor metabolism over the time between the PET scan and surgery, which may extend to more than $2 \mathrm{wk}(7,20)$.

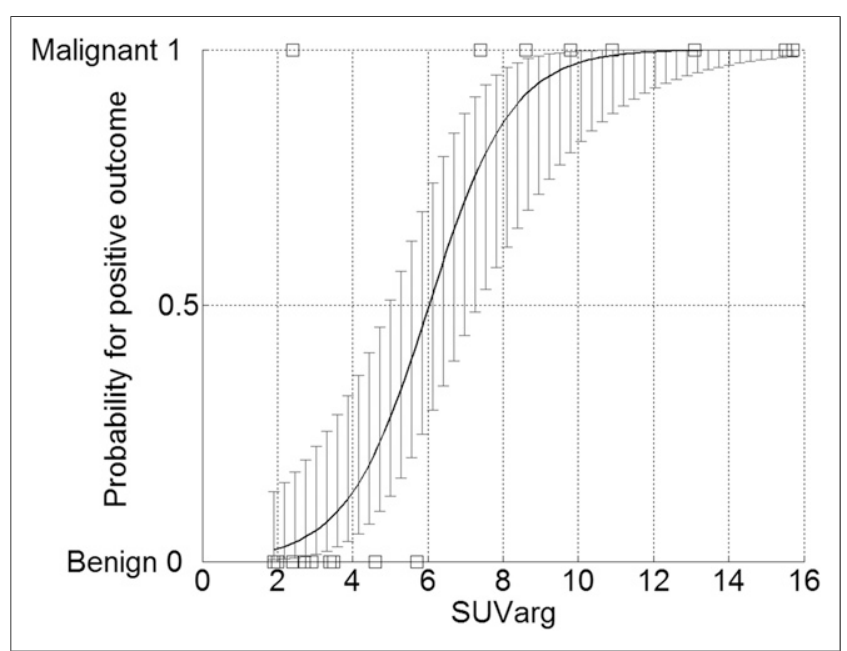

FIGURE 5. Liver biopsy outcome vs. specimen uptake. Error bars correspond to $95 \%$ confidence interval. 
TABLE 3

Comparison of SUV of Whole Specimens as Derived from Autoradiography and PET

\begin{tabular}{rrr}
\hline Patient no. & SUV $_{\text {ARG }}$ & SUV $_{\text {PET }}$ \\
\hline 3 & 9.1 & 9.2 \\
\hline 5 & 9.8 & 18.0 \\
\hline 7 & 4.3 & 3.7 \\
\hline 9 & 1.7 & 1.4 \\
10 & 15.5 & 7.2 \\
12 & 2.0 & 2.7 \\
\hline 14 & 6.1 & 3.4 \\
\hline 15 & 1.5 & 4.2 \\
\hline 16 & 2.4 & 2.4 \\
\hline 17 & 2.7 & 3.5 \\
\hline 18 & 8.6 & 6.4 \\
\hline 19 & 5.1 & 4.9 \\
\hline 20 & 8.6 & 7.8 \\
\hline 21 & 1.9 & 4.2 \\
\hline 22 & 3.1 & 3.5 \\
\hline 23 & 4.2 & 2.6 \\
\hline 24 & 2.3 & 3.2 \\
\hline 25 & 2.9 & 2.7 \\
\hline 26 & 5.7 & 7.6 \\
\hline 27 & 8.3 & 5.6 \\
\hline & & \\
\hline
\end{tabular}

In contrast, albeit only at the point of biopsy, QABS allows high spatial accuracy due to the visibility of the biopsy needle in the fused PET/CT image, pointing to the location of the extracted specimen in the tumor within minutes of the PET scan. For pathology sections of core specimens with preserved shape (i.e., not fragmented by pathology processing) and in the absence of motion between the CT and PET acquisitions, accuracy of less than $2 \mathrm{~mm}$ is achievable in fusing the autoradiography image and the pathology information with PET when the PET/CT scan is acquired with the needle in place. Because the biopsy needles usually protrude through the lesion boundary, the approach promises to provide unique information about border heterogeneity along the needle path.

The conclusions of the investigations using excised lesions are based on average results for a group of patients and are therefore useful for providing general guidance for cases similar to those investigated but are not reliable for indicating the location of the lesion border for any particular patient whose tumor was not excised. In contrast, QABS promises to provide individual-lesion information that can be used in the future management of the same patient and so adds an opportunity for personalizing cancer treatment.

With the QABS method, windowing of PET images can be refined and reconciled with smaller-scale autoradiography images and tissue-level histopathology correlation. Expanding this approach to a larger patient population may prospectively improve windowing accuracy for a broader tumor library. This is a step toward calibrating the PET image display window to indicate the lesion border as determined from pathology.

Recent studies have described tumor heterogeneity at different levels: histologic (e.g., vascularization), cellular, and genetic (21).
The impact of voxel heterogeneity on treatment outcome is not well understood and is currently under investigation (22). The high resolution of the autoradiography method is promising for investigating tracer distribution heterogeneities inside the biopsy specimen. Correlation of these observations with histopathology findings provides a unique opportunity for investigating the functional heterogeneity of the tumor. This information may be further correlated to the genetic profile of the tumor and to prognostic information.

The main challenges for transferring these correlations to the PET image are motion during imaging and the different spatial resolutions of the QABS method, histopathology, and PET. When breathing motion is problematic, bellows-assisted, monitored breath-holds $(23,24)$ and suspension of breathing with ventilation (25) may be used to reduce the registration error. The large size of the PET voxels compared with biopsy specimens may affect the autoradiography-PET comparisons in cases of nonuniform metabolic activity and requires more investigation. Despite that issue, along with the uncertainty factors discussed and the PET imaging artifacts (6), for most of the studied cases a correlation was observed between the activities determined by autoradiography and PET. With improvements in the QABS technique and in PET technology and resolution, the effect of these limitations is expected to decrease.

\section{CONCLUSION}

In situ, high-spatial-accuracy correlation of ${ }^{18} \mathrm{~F}-\mathrm{FDG}$ tracer uptake and histopathology findings is feasible by performing autoradiography of PET/CT-guided biopsy specimens. The information provided by QABS may affect various fields in oncology, including interventional radiology, pathology, radiation therapy, and nuclear medicine, in which studies correlating PET image features to tumor genotypic and phenotypic heterogeneity have yet to be systematically determined.

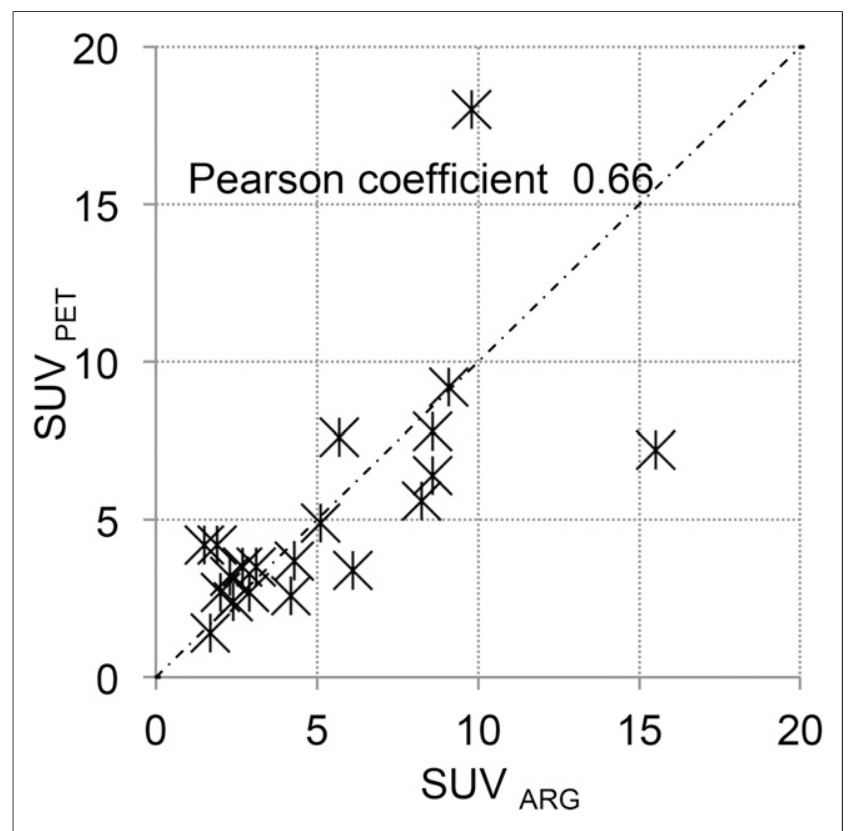

FIGURE 6. Correlation between PET and specimen uptake. 


\section{DISCLOSURE}

The costs of publication of this article were defrayed in part by the payment of page charges. Therefore, and solely to indicate this fact, this article is hereby marked "advertisement" in accordance with 18 USC section 1734 . This work was supported in part by a grant from Biospace Lab, S.A. No other potential conflict of interest relevant to this article was reported.

\section{ACKNOWLEDGMENTS}

We acknowledge the contribution of Dr. Anne Covey, Christian Czmielewski, and Polina Khersonskaya from Memorial SloanKettering Cancer Center.

\section{REFERENCES}

1. van Herk M. Margins and margin recipes. In: Palta JR, Mackie TR, eds. Uncertainties in External Beam Radiation Therapy. Madison, WI: Medical Physics Publishing; 2011:169-190.

2. MacManus M, Nestle U, Rosenzweig KE, et al. Use of PET and PET/CT for radiation therapy planning: IAEA expert report 2006-2007. Radiother Oncol. 2009;91:85-94.

3. Nestle U, Weber W, Hentschel M, Grosu AL. Biological imaging in radiation therapy: role of positron emission tomography. Phys Med Biol. 2009;54:R1-R25.

4. Boellaard R, O'Doherty MJ, Weber WA, et al. FDG PET and PET/CT: EANM procedure guidelines for tumour PET imaging: version 1.0. Eur J Nucl Med Mol Imaging. 2010;37:181-200.

5. Mawlawi O, Pan T, Macapinlac HA. PET/CT imaging techniques, considerations, and artifacts. J Thorac Imaging. 2006;21:99-110.

6. Boellaard R. Standards for PET image acquisition and quantitative data analysis. J Nucl Med. 2009;50(suppl 1):11S-20S.

7. Kirov AS, Fanchon LM. Pathology-validated PET image data sets and their role in PET segmentation. Clin Transl Imaging. 2014;2:253-267.

8. Solomon SB, Incorporating CT, MR imaging, and positron emission tomography into minimally invasive therapies. $J$ Vasc Interv Radiol. 2005;16:445-447.

9. Tatli S, Gerbaudo VH, Mamede M, Tuncali K, Shyn PB, Silverman SG. Abdominal masses sampled at PET/CT-guided percutaneous biopsy: initial experience with registration of prior PET/CT images. Radiology. 2010;256:305-311.
10. Tatli S, Gerbaudo VH, Feeley CM, Shyn PB, Tuncali K, Silverman SG. PET/CTguided percutaneous biopsy of abdominal masses: initial experience. $J$ Vasc Interv Radiol. 2011;22:507-514.

11. Cerci JJ, Neto CCP, Krauzer C, Sakamoto DG, Vitola JV. The impact of coaxial core biopsy guided by FDG PET/CT in oncological patients. Eur J Nucl Med Mol Imaging. 2013;40:98-103.

12. Shyn PB. Interventional positron emission tomography/computed tomography: state-of-the-art. Tech Vasc Interv Radiol. 2013;16:182-190.

13. Ryan ER, Sofocleous CT, Schoder H, et al. Split-dose technique for FDG PET/CTguided percutaneous ablation: a method to facilitate lesion targeting and to provide immediate assessment of treatment effectiveness. Radiology. 2013;268:288-295.

14. Azabdaftari G, Goldberg SN, Wang HH. Efficacy of on-site specimen adequacy evaluation of image-guided fine and core needle biopsies. Acta Cytol. 2010;54:132-137.

15. Nasuti JF, Gupta PK, Baloch ZW. Diagnostic value and cost-effectiveness of onsite evaluation of fine-needle aspiration specimens: review of 5,688 cases. Diagn Cytopathol. 2002;27:1-4

16. Higashi K, Clavo AC, Wahl RL. Does FDG uptake measure proliferative activity of human cancer cells? In vitro comparison with DNA flow cytometry and tritiated thymidine uptake. J Nucl Med. 1993;34:414-419.

17. Ryan ER, Thornton R, Sofocleous CT, et al. PET/CT-guided interventions: personnel radiation dose. Cardiovasc Intervent Radiol. 2013;36:1063-1067.

18. Deasy JO, Blanco AI, Clark VH. CERR: a computational environment for radiotherapy research. Med Phys. 2003;30:979-985.

19. Alessio A, MacDonald L. Spatially variant positron range modeling derived from CT for PET image reconstruction. In: Nuclear Science Symposium Conference Record. Piscataway, NJ: IEEE; 2008:3637-3640.

20. Caldas-Magalhaes J, Kasperts N, Kooij N, et al. Validation of imaging with pathology in laryngeal cancer: accuracy of the registration methodology. Int $J$ Radiat Oncol Biol Phys. 2012;82:e289-e298.

21. Gerlinger M, Rowan AJ, Horswell S, et al. Intratumor heterogeneity and branched evolution revealed by multiregion sequencing. $N$ Engl J Med. 2012;366:883-892.

22. Petit SF, Dekker AL, Seigneuric R, et al. Intra-voxel heterogeneity influences the dose prescription for dose-painting with radiotherapy: a modelling study. Phys Med Biol. 2009;54:2179-2196.

23. Shyn PB, Tatli S, Sainani NI, et al. Minimizing image misregistration during PET/CT-guided percutaneous interventions with monitored breath-hold PET and CT acquisitions. J Vasc Interv Radiol. 2011;22:1287-1292.

24. Shyn PB, Tatli S, Sahni VA, et al. PET/CT-guided percutaneous liver mass biopsies and ablations: targeting accuracy of a single $20 \mathrm{~s}$ breath-hold PET acquisition. Clin Radiol. 2014;69:410-415.

25. Li G, Burger IA, Schmidtlein CR, Ridge C, Solomon SB, Humm JL. Liver tumor volume determination by PET using ventilator suspended respiration [abstract]. Med Phys. 2013;40(suppl):105. 